CASE REPORT

\title{
The squirting bleb: image and treatment of inspiration- linked leakage of a peritoneopleural fistula
}

\author{
F H Krouwels, P Bresser
}

Thorax 2004;59:353-354. doi: 10.1136/thx.2003.005488

\begin{abstract}
A 25 year old man with known chronic right sided heart failure and ascites due to a congenital heart defect presented with dyspnoea and a massive pleural effusion. Thoracoscopy revealed two diaphragmatic blebs. Changes in peritoneal and thoracic pressure during respiration resulted in periodic squirting of a ruptured bleb, illustrating preferential flow of peritoneal fluid into the thorax. The pleural effusion was successfully treated with drainage of ascitic fluid and chemical pleurodesis.
\end{abstract}

A 25 year old man was referred to our clinic with gradually increasing exertional dyspnoea. He was known to have a congenital univentricular heart for which he had had a corrective Fontan operation in 1980. He had since suffered from chronic right sided heart failure resulting in a protein losing enteropathy and ascites which was treated with diuretics and a diet of medium chain triglycerides.

On presentation he was severely dyspnoeic, had gained $7 \mathrm{~kg}$ in weight, and his abdomen was enlarged. Chest radiography revealed a massive left sided pleural effusion and a large amount of ascitic fluid was seen on ultrasound examination. He also had raised venous pressure and hypoalbuminaemia.
The patient was treated with tube drainage of the pleural fluid and diuretics; 4 litres of pleural fluid with characteristics of a transudate (albumin $3 \mathrm{~g} / \mathrm{l}$, lactate dehydrogenase (LDH) $34 \mathrm{U} / \mathrm{l}$; serum levels: albumin $15 \mathrm{~g} / \mathrm{l}$, LDH $212 \mathrm{U} / \mathrm{l}$ ) were removed. Because of persistent pleural fluid production of more than $0.5 \mathrm{l} /$ day, an inspection thoracoscopy was performed. A tense bleb was seen on the diaphragm and another bleb was visible which was ruptured and forcefully squirted fluid into the thoracic cavity when the patient inspired (fig 1). No other defects were observed. For further drainage a pleural tube was left in place, and a peritoneal tube was inserted to minimise the pressure on the diaphragm and the flow over the fistula. The next day the pleural tube leakage had nearly stopped and the chest radiograph showed only a small pleural effusion. Chemical pleurodesis was then performed with quinacrine (100 mg on three consecutive days) and the pleural drain was removed. Ten days after the removal of the peritoneal tube the patient was discharged in relatively good health without dyspnoea. After 2 years there are no clinical or radiological signs of recurrence of the pleural effusion.

\section{DISCUSSION}

Fontan operations for congenital heart abnormalities are frequently complicated by chronically raised systemic venous pressure. This can result in hepatomegaly, ascites and protein losing enteropathy. ${ }^{1}$ Pleural effusions in such patients can be
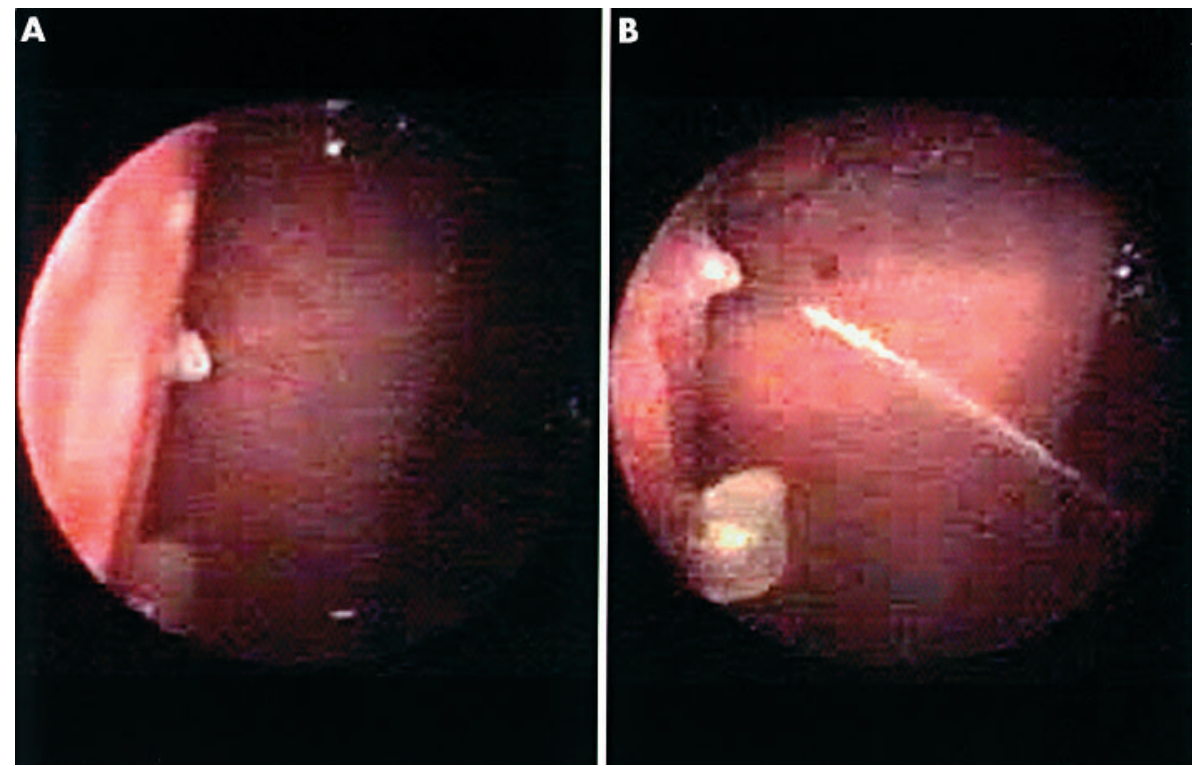

Figure 1 Thoracoscopic image of the diaphragmatic blebs: the diaphragm is situated on the left side in each figure. (A) At rest and during expiration the upper lacerated bleb is nearly collapsed while the lower bleb is undamaged and tense. (B) At inspiration the lacerated bleb forcefully squirts fluid into the thoracic cavity. 
the result of heart failure, hypoalbuminaemia, impaired lymphatic drainage, or leakage of ascites through the diaphragm. Unilateral left sided effusions are seldom seen. The venous pressure and albumin concentration in this patient on presentation were similar to those of the previous years but a large amount of pleural fluid was produced daily. We therefore hypothesised that the pleural effusion was caused by leaking of peritoneal fluid through the diaphragm. This has been described in patients with ascites due to liver cirrhosis, ${ }^{2}$ in patients with peritoneal dialysis, ${ }^{3}$ and those with ovarian hyperstimulation syndrome. ${ }^{4}$ In these patients diaphragmatic defects or blebs have been found and it was postulated that peritoneal fluid could pass through them into the pleural cavity. Such passage can be shown with dyes or by nuclear imaging. In our patient thoracoscopy showed transdiaphragmatic leakage during inspiration caused by a ruptured diaphragmatic bleb forcefully squirting peritoneal fluid into the thorax. This shows how respiratory movements induce a transdiaphragmatic pressure gradient resulting in preferential flow of fluid from the peritoneum into the thoracic cavity. Such a mechanism has been hypothesised but has never been shown, and explains how only a small amount of peritoneal fluid results in a clinically significant pleural effusion. ${ }^{4}$

Several procedures have been described to treat peritoneopleural fistulas. Clearly, the most efficacious treatment is ligation of the blebs (either by videothoracoscopy or thoracotomy) in combination with talc pleurodesis. This procedure was successful in $60 \%$ of 18 patients with a hepatic hydrothorax while talc insufflation alone was successful in $44 \%$ of patients. ${ }^{5}$ The combination of videothoracoscopic surgery and talc pleurodesis was successful in all five patients with a peritoneopleural fistula due to peritoneal dialysis. ${ }^{6}$
There are no studies reported of patients with right sided heart failure. As the cardiovascular status of our patient did not permit general or spinal anaesthesia, we used a combination of ascites drainage and chemical pleurodesis which resulted in satisfactory healing of the fistula and this persists to the present time.

\section{Authors' affiliations}

P Bresser, Department of Pulmonology, Academic Medical Center, University of Amsterdam, The Netherlands

F H Krouwels, Department of Pulmonology, OLVG Hospital, 1090 HA Amsterdam, The Netherlands

Correspondence to: Dr F H Krouwels, Department of Pulmonology, OLVG Hospital, PO Box 95500, 1090 HA Amsterdam, The

Netherlands; F.H.Krouwels@olvg.nl

Received 16 February 2003

Accepted 5 February 2004

\section{REFERENCES}

1 Girod $A D$, Fontan $F$, Deville $C$, et al. Long-term results after the Fontan operation for tricuspid atresia. Circulation 1987;75:605-10.

2 Chen A, Ho YS, Tu YC, et al. Diaphragmatic defect as a cause of massive hydrothorax in cirrhosis of liver. J Clin Gastroenterol 1988;10:663-6.

3 Adam WR, Arkles LB, Gill G, et al. Hydrothorax with peritoneal dialysis: radionuclide detection of a pleuroperitoneal connection. Aust NZ J Med $1980 ; 10: 330-2$.

4 Loret de Mola JR. Pathophysiology of unilateral pleural effusions in the ovarian hyperstimulation syndrome. Hum Reprod 1999;14:272-3.

5 De Campos JRM, Andrade Filho LO, de Campos Werebe E, et al. Thoracoscopy and talc poudrage in the management of hepatic hydrothorax. Chest 2000;1 18:13-7.

6 Jagasia MH, Cole FH, Stegman MH, et al. Video-assisted talc pleurodesis in the management of pleural effusion secondary to continuous ambulatory peritoneal dialysis: a report of three cases. Am J Kidney Dis 1996;28:772-4.

\section{LUNG ALERT}

\section{Non-linear relationship between lung cancer risk and cigarette tar yield}

$\Delta$ Harris JE, Thun MJ, Mondul AM, et al. Cigarette tar yields in relation to mortality from lung cancer in the cancer prevention study II prospective cohort, 1982-8. BMJ 2004;328:72-6

- his study was undertaken to determine the risk of lung cancer associated with medium, low, and very low tar filter cigarettes. Using a cohort of patients from the US cancer prevention study II (CPS-II), 364239 men (100868 current smokers) and 576535 women (124 270 current smokers) were studied. The primary outcome measure was death from tracheal or lung cancer over 6 years of follow up. Tar rating per cigarette brand was designated high ( $\geqslant 22 \mathrm{mg}$ ), medium ( $15-21 \mathrm{mg})$, low (8-14 mg), or very low ( $\leqslant 7 \mathrm{mg}$ ). Assessment was made using Cox proportional hazards analysis and adjustment made for multiple covariates.

The risk of lung cancer was increased in current smokers, with high tar cigarette smokers experiencing the highest risk. No difference was evident between the medium, low, and very low tar brands. These results withstood sensitivity analysis, including exclusion of people with smoking related diseases. The study results challenge the assumption of a linear association between tar rating and lung cancer risk. "Compensatory smoking" in those smoking lower tar brands (deeper inhalation with increased puff volume, longer duration of smoke retention, and a greater total number of cigarettes smoked) and the poor correlation between tar yield and carcinogen delivery offer possible explanations. Cessation should be advised in all smokers and patients informed that low tar cigarettes are not a safer alternative.

H E Davies 